\title{
Linear Time Varying Precoder Applied to an ISI Channel
}

\author{
Nader Sheikholeslami and Peter Kabal \\ Department of Electrical Engineering \\ McGill Universit y,Montreal, Quebec, Canada \\ nader@ee.mcgill.ca \\ kabal@ee.mcgill.ca
}

\begin{abstract}
A linear time varying precoding structure for data transmission over ISI channels is investigated. This precoding sc heme is capable of stabilizing the in erse channel filter without increasing the dynamic range of the receiv ed information symbols. This is particularly useful if the receiver decision device is a simple slicer with fixed number of levels.
\end{abstract}

\section{INTRODUCTION}

For fast digital transmission over bandlimited channels, precoding at transmitter can be used to con trol intersymbol interference (ISI). By performing channel equalization at the transmitter, this arrangements a voids noise enhancement, as in linear equalizer at the receiver, and avoids error propagation, as in a decision feedback equalizer. How erer, a straigh tformard prefiltering can boost the transmit signal pow er.

Tomlinson-Hanshima Precoding (THP) is a practical solution to eliminate ISI and limit the peak and average of transmit pow er [1] [2].THP performs modulo arithmetic to limit the precoded signal to a preset maximum and minimum levels. There is a serious drawback associated with THP; it increases the dynamic range of the received signal. This increase in dynamic range can be particularly severe if the spectrum of the channel contains spectral nulls. If the original symbols are tak en from a finite set, THP will increase the size of this set for the received signal. A modulo cater is usually in place prior to the decision device at receiver to a void increasing the mumber of slicer levels.

In some applications however, the decision device at the receiver uses a simple slicer with fixed number of decision levels. One such application is high speed data transmission over PCM voiceband channels [3] [4]. In PCM voiceband channel usually the only analog portion of the netw ork is the subscriber loop connecting an end user to the central office (Fig. 1). There are also users with direct digital connection to the network (e.g. in ternet service providers with $\mathrm{T} 1$ links to the switched network).

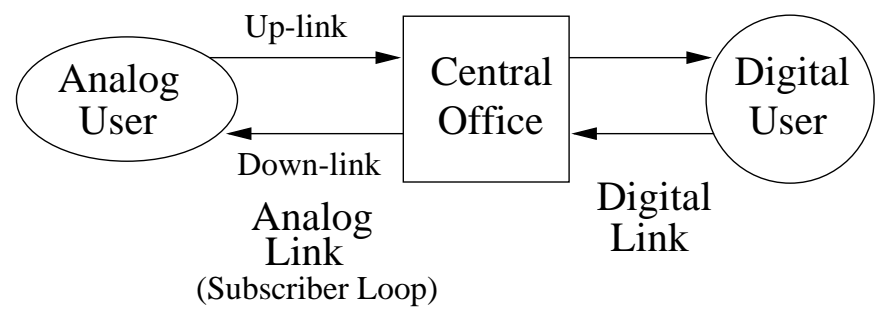

Fig. 1 Analog and digital links to Central Office

The predominant source of noise for a PCM channel is quantization error due to Analog-to-Digital (A/D) con ersion. How erer, the down-link connection from a digitally connected user to a user connected to subscriber loop does not undergo an $\mathrm{y} / \mathrm{D}$ con version. Therefore, the maximum data rate on this channel can theoretically reach $64 \mathrm{kbit} / \mathrm{s}$ (assuming $8 \mathrm{kHz}$ sampling rate and 256 levels for transmit signal). In fact this idea has been recently used to develop $56 \mathrm{~kb} / \mathrm{s}$ modems.

In this paper we consider the up-link channel where quantization error occurs. T oeliminate the quantization error, the signal at the A/D at each sampling instance should take values equal to one of the quantizer levels. This, of course, requires synchronization betw een transmitter and A/D. Hov ev er, synhronization is not the only concern for this transmission channel. An anti-aliasing filter prior to A/D reduces the bandwidth of the channel. F or instance, in a PCM dhannel with $8 \mathrm{kHz}$ sampling rate the discrete model of the channel includes nulls at DC and a reduced response in the $3.5-4 \mathrm{kHz}$ band. Since the number of quantization levels is fixed, any increase in the dynamic range of received signal would reduce the number of useful quantizer levels or would cause ov erload errors. Therefore, THP is not appropriate choice of precoding for this channel. 
We discuss an alternative precoding approach which does not increase the dynamic range of the received information symbols. By introducing redundancy among the data symbols, we perform spectral shaping of the input spectrum. As a result we can control the transmit signal power.

\section{PRELIMINARIES}

\subsection{Channel Model}

Figure 2 shows the discrete time model of the channel. In this model we assume $h(n)$ is a monic, FIR filter. $h(n)$ represents the effect of the subscriber loop and the anti-aliasing filter prior to $\mathrm{A} / \mathrm{D}$ at the receiver. Since subscriber loops usually have transformer coupling, there is a spectral null at DC.

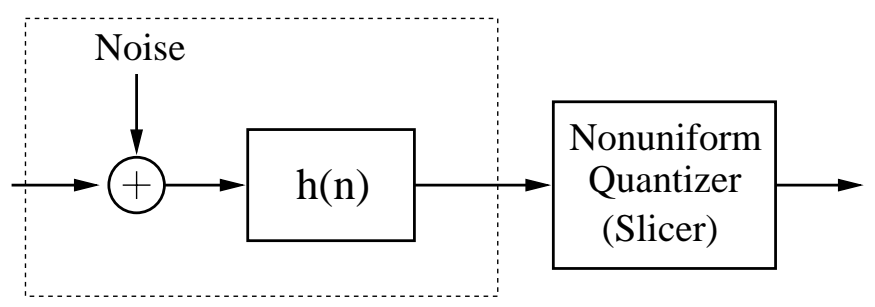

Channel

Fig. 2 Discrete time model of the channel and receiver

The additive noise in this channel is due to crosstalk from other users and thermal noise. A non-uniform quantizer (i.e A-law or $\mu$-law) is in place to discretize the output of the channel. In this model, the quantizer serves as the decision device (slicer in communication sense). Therefore, appropriate signaling over this channel would be a baseband PAM transmission with non-uniform distribution of levels (the same as that of non-uniform quantizer)

\subsection{Modulo arithmetic Precoding}

The channel filter, $h(n)$ introduces intersymbol interference to received signal. If $h(n)$ is known at the transmitter, the transmit signal can be precoded using the inverse filter $1 / H(z)$. But having any roots of $H(z)$ with magnitude greater or equal to unity results in an unstable inverse filter. Employing modulo arithmetic, Tomlinson-Harashima Precoder (THP) limits the output of the inverse filter to a preset maximum and minimum levels to effectively "stabilize" the system.

The operation of THP can be interpreted as a nonlinear time varying system as shown in Fig 3 . For input signal taking real values in $\left(-V_{\max } / 2,+V_{\max } / 2\right]$ interval, a unique integer multiple of $V_{\max }$ is added to $x(n)$ to limit the output values of the inverse filter to the same interval. Therefore the effective input to the inverse filter is :

$$
s(n)=x(n)+V_{\max } \cdot k(n)
$$

where $k(n)$ is an integer. The Modulo Operator is a nonlinear, memoryless function which extends the input signal as stated in (1). The extension of input signal directly affects the dynamic range of the channel output. To detect $x(n)$ at the receiver, a modulo operator should be in place to subtract $V_{\max } \cdot k(n)$ from each detected symbol. It is worth noting that while THP limits the transmit signal power, due to possibly large values of $k(n)$, it increases the dynamic range of the received signal. This problem is more severe for ISI channels with spectral nulls. Since in our channel model the decision device uses a simple slicer, using THP would reduce the effective number of slicer levels or cause overload errors.

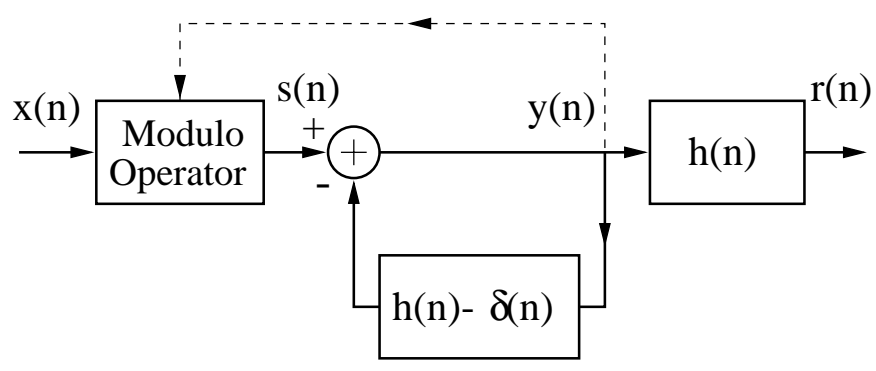

Fig. 3 Tomlinson-Harashima Precoder

\section{LINEAR TIME VARYING PRECODING}

We consider an alternative approach to control the peak power of the transmit signal without increasing the dynamic range of the received information symbols. In order to stabilize the inverse filter $1 / H(z)$, we add redundancy to the input signal to cancel poles of the inverse filter. In another words, spectral shaping of the input signal will cause pole-zero cancellation and results in a stable system.

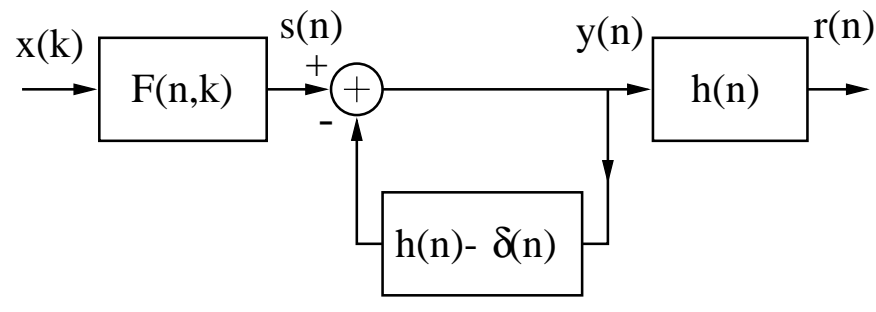

Fig. 4 Linear Time Varying Precoder 
Figure 4 shows the proposed structure of the transmitter. The inverse filter, $1 / H(z)$, is realized using a direct form structure with a feedback connection. $F(n, k)$ is a linear time varying operator which upsamples the input. We assume $F(n, k)$ operates on a block of $(N-M)$ inputs and creates $N$ outputs where $M$ is the memory length of the channel (smaller than the output block length, $N$ ). The added symbols are a linear combination of the previous input values which result in spectral shaping of $S(z)$, the $z$-transform of $s(n)$ :

$$
S(z)=H(z) \cdot Y(z)
$$

where $Y(z)$ is the output signal. $S(z)$ can be decomposed into non-overlapping blocks of length $N$ as follows:

$$
\begin{aligned}
S(z) & =\sum_{n=-\infty}^{\infty} s(n) z^{-n} \\
& =\sum_{m=-\infty}^{\infty} \sum_{l=0}^{N-1} s(m N+l) z^{-(m N+l)} \\
& =\sum_{m=-\infty}^{\infty}\left(\sum_{l=0}^{N-1} s(m N+l) z^{-l}\right) z^{-m N} \\
& =\sum_{m=-\infty}^{\infty} S_{m}(z) z^{-m N}
\end{aligned}
$$

To make $H(z)$ a factor of $S(z)$, it is sufficient that:

$$
S_{m}(z)=H(z) \cdot Y_{m}(z)
$$

$H(z)$ can be written as:

$$
H(z)=\sum_{k=0}^{M} h(k) z^{-k}
$$

Based on (4) and (5), $Y_{m}(z)$ is a polynomial of degree $(N-M-1)$ :

$$
Y_{m}(z)=\sum_{k=0}^{N-M-1} y_{m}(k) z^{-k}
$$

Equation (4), applies $M$ conditions among $S_{m}(z)$ coefficients but $S_{m}(z)$ contains $N$ unknown values. Therefore, there is more than one solution for $S_{m}(z)$ (note that $N>M$ ). One possible way to determine coefficients $s_{m}(l)$ is the following:

Let the first $(N-M)$ values of $s_{m}(l)$ in each block be the same as inputs:

$$
\begin{aligned}
& s_{m}(l)=x(m(N-M)+l) \\
& \quad \text { for } l=0, \cdots, N-M-1
\end{aligned}
$$

Extending the inverse filter as a causal sequence, we have:

$$
1 / H(z)=\sum_{k=0}^{\infty} h_{i n v}(k) z^{-k}
$$

By replacing (8) in (4), $y_{m}(l)$ is determined as:

$y_{m}(l)=\sum_{k=0}^{l} h_{i n v}(k) \cdot s(m N+l-k)$ for $0 \leq l<N-M$

Therefore the last $M$ values of $s_{m}(l)$ are:

$$
\begin{aligned}
& s_{m}(l)=\sum_{k=0}^{M} h(k) \cdot y_{m}(l-k) \\
& \quad \text { for } \quad N-M \leq l<N
\end{aligned}
$$

where $y_{m}(l)$ is zero padded as follows:

$$
y_{m}(l)=0 \text { for } N-M \leq l<N
$$

Equations (7) and (10) totally specify the inverse filter input, $s(n)$.

The output of precoder, $y(n)$ is determined using (9) and (11). It is worth noting that since $y_{m}(l)$ is a finite sum of bounded values, $y(n)$ is also bounded.

The above discussion provides a theoretical justification for applying linear time varying precoding. In this precoding scheme, the prefilter makes the channel transparent for data symbols and extra symbols added to the original data stabilize the prefilter.

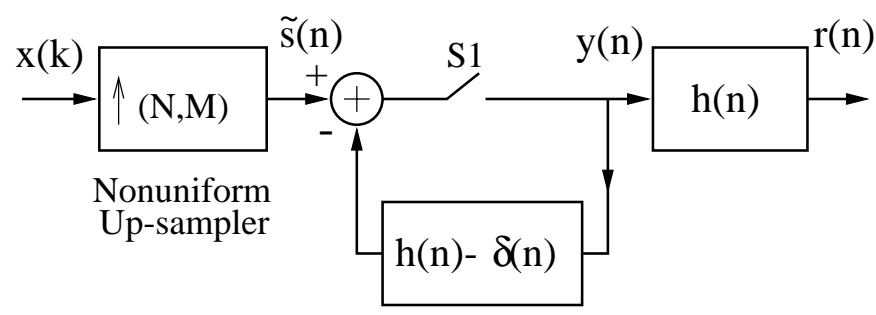

Fig. 5 A simple implementation of Time Varying Precoder

Figure 5 shows an alternative implementation of the same time varying precoder. The non-uniform upsampler block increases the symbol rate of the inputs by adding $M$ zeros to the end of each block of $(N-M)$ input symbols. S1 performs as an on-off switch with a pattern of $(N-M)$ ones followed by $M$ zeros. By turning off $\mathrm{S} 1$ for $M$ consequent symbols, we clear the memory of the inverse filter. Therefore after each block of $N$ symbols the precoder starts from zero initial state. The output of the precoder for each block of length $N$ can be written as: 


$$
\begin{aligned}
& y(m N+l)= \\
& \begin{cases}\sum_{k=0}^{l} \tilde{s}(m N+l-k) \cdot h_{i n v}(k) & 0 \leq l<N-M \\
0 & N-M \leq l<N\end{cases}
\end{aligned}
$$

where $h_{i n v}(n)$ is causal impulse response corresponding to the inverse filter $1 / H(z)$ as defined in (8). The inverse filter impulse response, $h_{i n v}(n)$ may tend to infinity for large values of $n$ but S1 truncates the impulse response and only the first $(N-M)$ values of $h_{i n v}(n)$ contribute to precoder output. Therefore, the output of precoder remains stable. It can be shown that the channel output, $r(n)$ is identical to the input signal for the first $(N-M)$ values in each block.

For a block length $N$ much larger than the channel memory, the information rate reduction is tolerable. In the case of channel with long memory, $h(n)$ can be decomposed into two parts:

$$
h(n)=h_{1}(n) * h_{2}(n)
$$

where $H_{1}(z)$ has roots well inside the unit circle so the inverse of $H_{1}(z)$ is always stable. $H_{2}(z)$ contains the remaining roots including those outside or on the unit circle. The linear time varying precoder is applied to $h_{2}(n)$ followed by an inverse filter compensating for $H_{1}(z)$.

\section{EXAMPLE}

As an example the following channel is considered:

$$
\begin{aligned}
H(z)= & \left(1-z^{-1}\right)\left(1+z^{-1}\right) \\
& \times\left(1-e^{j 0.8 \pi} z^{-1}\right)\left(1-e^{-j 0.8 \pi} z^{-1}\right)
\end{aligned}
$$

which has four zeros on the unit circle. We used the time varying precoder to compensate this channel. A random input sequence was applied to the precoder. Figure 6 shows the transmit power versus normalized information symbol rate defined as:

$$
R=\frac{N-M}{N}
$$

where $M$ is the memory length of the channel and $N$ is the block length. Increasing the data symbol rate increases the transmit power exponentially. As shown in (12), the precoder output at each time instance is a linear combination of its input with coefficients $h_{i n v}(k)$. By increasing the length of each block each output can take larger value (note that the inverse filter is unstable and running the summation for large values of $l$ in (12) results in an unbounded output). The choice of $N$ depends on maximum allowable transmit power.

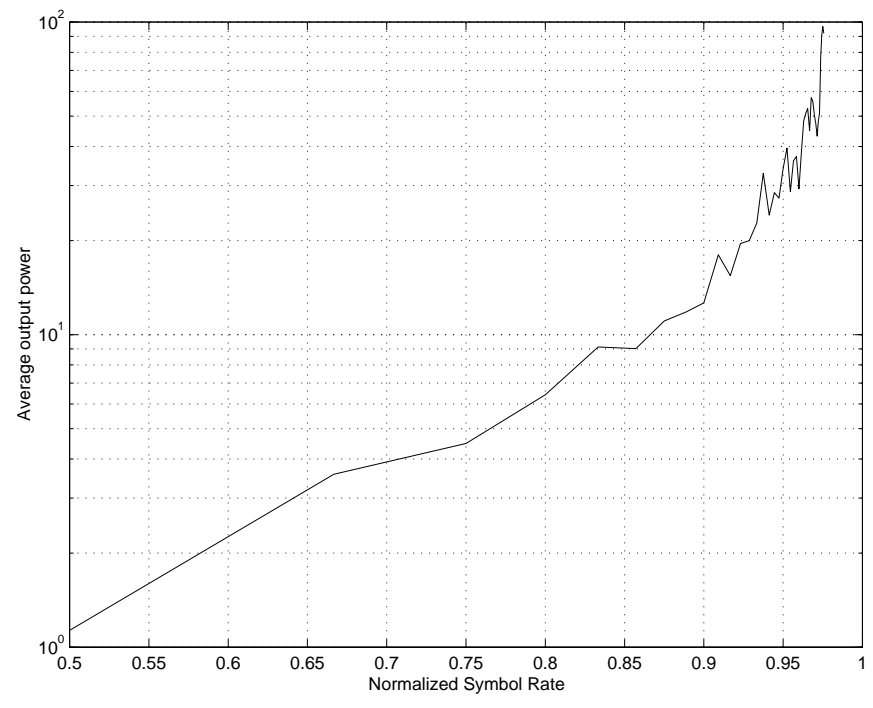

Fig. 6 Average output power vs. normalized symbol rate

\section{CONCLUSION}

We have investigated a precoding scheme to equalize a channel at the transmitter without increasing the dynamic range of the received signal. We developed a theoretical ground for linear time varying precoding. Compared to THP, this time varying precoding does not change the data symbol values to control the output power, instead it adds appropriate redundancies to the input signal to cancel undesired poles of the prefilter. The price to be paid for stabilizing the system is a reduction of the maximum rate. A simple implementation of the time varying precoder employs a single switch in the feedforward path of the prefilter. Using an example the trade off between maximum information rate and average power would be seen.

\section{REFERENCES}

[1] E. A. Lee and D. G. Messerschmitt, Digital Communication. KAP, 1994.

[2] M. Tomlinson, "New automatic equaliser employing modulo arithmetic," Electronics Letters, vol. 7, pp. 138-139, March 1971.

[3] I. Kalet and J. E. Mazo and B. R. Saltzberg, "The capacity of PCM voiceband channels," Proc. IEEE Int. Conf. Communications, pp. 507-511, 1993.

[4] P. A. Humblet and M. G. Troulis, "The information driveway," IEEE Communications Magazine, vol. 34, pp. 64-68, December 1996. 